\title{
Parâmetros reprodutivos do curimbatá no rio Mogi-Guaçu
}

\author{
Roseli Ormanezi Ramos ${ }^{1}$, Alberto Carvalho Peret ${ }^{2}$, Sergio Moreira Ramos ${ }^{1}$, José Sávio Colares de Melo
}

\section{RESUMO}

Com o objetivo de obter informações sobre parâmetros da biologia reprodutiva da população de curimbatá, visando ao gerenciamento da espécie voltado para sua conservação, foram realizadas coletas mensais de peixes no período de fevereiro de 2003 a setembro de 2004 em dois pontos do rio Mogi-Guaçu. Foram capturados 176 fêmeas e 140 machos. Os parâmetros analisados foram relação peso-comprimento, fator de condição (K), índice gonadossomático (IGS), proporção sexual e abundância relativa. Os indivíduos adultos de curimbatá do rio Mogi-Guaçu têm crescimento alométrico negativo. Os maiores valores do fator de condição (K) e de IGS ocorreram durante o período reprodutivo, quando a proporção sexual foi de 1:1. O período de reprodução de curimbatá ocorre entre os meses de outubro e janeiro, com picos em novembro e dezembro. Os machos de curimbatá apresentam antecipação da maturação gonadal em relação às fêmeas.

Palavras-chave: crescimento, fator de condição, índice gonadossomático, Prochilodus lineatus, proporção sexual.

\section{ABSTRACT}

\section{Reproductive parameters of curimbatá (Prochilodus lineatus) in the Mogi-Guaçu river}

The objective of this work was to generate information about reproductive biology parameters of a curimbatá population for use in managing the species toward its conservation. Fish were monthly collected from February 2003 to September 2004 in two sites of the Mogi-Guaçu river. A total of 176 females and 140 males were captured. The parameters weight-length relationship, condition factor (K), gonad-somatic index (GSI), sex ratio and relative abundance were analyzed. Curimbatá adults from the Mogi-Guaçu river showed negative allometric growth. The highest values of condition factor $(\mathrm{K})$ and GSI occurred during the reproductive period, when the sex ratio was 1:1. The period of curimbatá reproduction occurs between October and January, with peaks in November and December. Curimbatá males show anticipation of gonadal maturation in relation to females.

Key words: Condition factor, growth, gonad-somatic index, Prochilodus lineatus, sexual proportion.

\footnotetext{
Recebido para publicação em março de 2008 e aprovado em junho de 2010

${ }^{1}$ Biólogo, Doutor. Centro Nacional de Pesquisa e Conservação de Peixes Continentais - CEPTA/ICMBio, Rodovia Pref. Euberto Nemésio Pereira de Godoy, km 6,5, Caixa Postal 64 -13.630-970, Pirassununga, São Paulo, Brasil. roseli.ramos@ibama.gov.br; sergio.ramos@ibama.gov.br

${ }^{2}$ Biólogo, Doutor. Universidade Federal de São Carlos - UFSCar, Rodovia Washington Luiz, Km 235, Monjolinho, Caixa Postal 676, 13.565-905, São Carlos, São Paulo, Brasil. peret@power.ufscar.br

${ }^{3}$ Engenheiro de Pesca, Doutor. Centro Nacional de Pesquisa e Conservação de Peixes Continentais - CEPTA/ICMBio, Rodovia Pref. Euberto Nemésio Pereira de Godoy, km 6,5, Caixa Postal 64 - 13.630-970, Pirassununga, São Paulo, Brasil. jose-savio.melo@ibama.gov.br
} 


\section{INTRODUÇÃO}

O curimbatá, peixe de piracema e com hábito alimentar iliófago, realiza migrações reprodutivas para montante e trófica para jusante no rio Mogi-Guaçu e é o de maior frequência na bacia superior do rio Paraná, envolvendo os rios Grande, Pardo e Mogi-Guaçu (Godoy, 1975). No entanto, segundo Morelli et al. (2007), embora essa espécie realize grandes migrações durante seu ciclo de vida, cardumes residentes são encontrados o ano todo na Barragem de Cachoeira de Emas.

Diferentes parâmetros têm sido avaliados em estudos de dinâmica de população, dentre eles a relação peso-comprimento e o fator de condição (Braga, 1986; Barbieri \& Verani, 1987; Araujo et al., 2000; Gomieiro \& Braga, 2003; Gurgel, 2004; Orsi et al., 2004). O fator de condição fornece informações sobre o estado de bem-estar do peixe no ambiente em que vive (Vazzoler, 1996) e deve permanecer constante, independentemente do tamanho que o peixe possa vir a ter em determinado período (Braga, 1986). Variações no fator de condição podem estar associadas ao acúmulo de gorduras, que por sua vez pode interferir com o período de desenvolvimento gonadal e concomitantemente com o processo reprodutivo dos curimbatás (Barbieri \& Verani, 1987). Conforme Gurgel et al. (1997), o fator de condição pode sofrer alterações em função de fatores extrínsecos (reservas orgânicas, desenvolvimento gonadal e tamanho dos exemplares) e extrínsecos (disponibilidade alimentar, temperatura, fotoperíodo, entre outros).

A relação peso-comprimento fornece informações sobre o tipo de crescimento da espécie, podendo ser isométrico ou alométrico. Segundo Orsi et al. (2002), se o coeficiente b for igual a 3, o crescimento será isométrico; se for maior que 3, será alométrico positivo, e se for menor que 3, será alométrico negativo. Esses autores acrescentam ainda que se o crescimento for isométrico, o incremento em peso acompanha o crescimento em comprimento, mas se for alométrico negativo, há um incremento em peso menor do que em comprimento; e se é alométrico positivo, há um incremento em peso maior do que em comprimento. Segundo Gurgel (2004), o parâmetro b, assim como as variáveis peso e comprimento, pode também variar para peixes de mesma espécie, mas dentro de alguns limites, de acordo com a localidade, o comprimento médio da população e a idade.

Dessa forma, é necessário avaliar parâmetros da biologia reprodutiva da população do curimbatá, Prochilodus lineatus, observados na barragem de Cachoeira de Emas e na foz do rio Jaguari-Mirim.

\section{MATERIAL E MÉTODOS}

A espécie utilizada neste experimento foi o curimbatá (Prochilodus lineatus Valenciennes, 1836).

Os exemplares adultos foram capturados mensalmente no período de fevereiro de 2003 a setembro de 2004 em dois pontos do rio Mogi-Guaçu, sendo o ponto 1 localizado na Barragem de Cachoeira de Emas, Pirassununga, São Paulo, e o ponto 2 distando $13 \mathrm{~km}$ à montante, na foz do Jaguari-Mirim, Pirassununga, São Paulo, nas coordena-

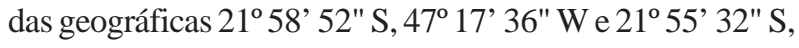
$47^{\circ} 21^{\prime} 59^{\prime \prime} \mathrm{W}$, respectivamente.

Para a captura dos exemplares foram utilizadas tarrafas de malha 11 cm entre nós opostos medida com malha esticada.

Após a captura, os exemplares foram acondicionados em caixas com capacidade de 200 L, com água e suplementação de oxigênio e transportados para o laboratório de reprodução do CEPTA/IBAMA/Pirassununga para serem amostrados.

Com o auxílio de um ictiômetro com precisão de $1 \mathrm{~mm}$ foram medidos o comprimento total (cm), tomando-se as medidas entre as extremidades anterior e posterior do animal, e o peso total (g) com o auxílio de balança analítica, com precisão de 0,001 g. Em seguida, foi aberta a cavidade abdominal, e as gônadas foram observadas in situ. Uma vez afastadas as vísceras, as gônadas foram analisadas quanto ao aspecto macroscópico (tamanho) segundo Godoy (1975) e retiradas cuidadosamente para pesagem em balança analítica, com precisão de 0,001 g.

A relação peso-comprimento foi determinada para verificar o tipo de crescimento da espécie. Os valores foram lançados em gráfico de dispersão, considerando-se como variável dependente o peso total e independente, o comprimento total.

Foram obtidas as expressões das curvas das relações peso-comprimento para machos e para fêmeas, de cada ponto de coleta, por meio da equação proposta por Le Cren (1951). Foi aplicado o teste t de Student aos dados do parâmetro $b$ das expressões peso-comprimento para estabelecer o tipo de crescimento (isométrico ou alométrico).

Para a determinação do fator de condição (Kn) e do índice gonadossomático (IGS), foram utilizadas as expressões das equações adotadas por Vazzoler (1996).

Para a caracterização macroscópica das gônodas, foi utilizada a escala adotada por Godoy (1975) modificada, considerando cinco estádios de maturidade: repouso, em maturação, parcialmente maduro, maduro e esgotado.

A proporção sexual foi determinada pelas frequências mensais de ocorrência de machos e fêmeas coletados. Aplicou-se o teste não paramétrico G (Sokal \& Rohlf, 1981), a 5\% de significância, para detectar possíveis diferenças significativas nessas proporções.

A abundância relativa para o curimbatá foi expressa em captura por unidade de esforço (CPUE), sendo observadas as variações temporal (meses) e espacial (local) de fevereiro de 2003 a setembro de 2004. A relação expressa o peso total (g) do peixe capturado pelo número de lances de tarrafas:

\section{$\mathrm{CPUE}=\mathrm{Wt} / \mathrm{N}^{\circ}$}




\section{RESULTADOS E DISCUSSÃO}

A relação entre peso e comprimento foi estabelecida para 316 exemplares adultos, sendo 176 fêmeas e 140 machos.

Não houve diferenças significativas $(\mathrm{p}>0,05)$ para o parâmetro b entre os sexos em cada ponto de coleta e entre eles. $\mathrm{O}$ crescimento dos peixes é do tipo alométrico negativo, apresentando maior incremento em comprimento do que em peso $(b<3,0)$ (Figura 1). Os valores da constante $b$ situaram-se dentro dos limites indicados por Vazzoler (1996), que varia de 2,4 a 4,0 para a maioria das espécies de peixes. Goulart \& Verani (1992) encontraram b = 2,81 para Hypostomus commersonii; Vianna \& Verani (2002), b = 3,97 para Orthopristis ruber; Costa et al. (2005), b = 2,79 para Leporinus copelandii; e Gurgel (2004), b = 2,59 para Astyanax fasciatus. Pequenas variações em torno desse parâmetro podem ser reflexos de diferenças ambientais e aspectos biogenéticos inerentes a cada espécie (Verani, 1980).

De acordo com Barbieri et al. (2000), que também trabalharam com curimbatá na barragem de Cachoeira de Emas, essa espécie apresenta crescimento isométrico. Os autores sugerem ainda que a população de curimbatá do rio Mogi-Guaçu está iniciando o processo reprodutivo com tamanho e idade inferiores (precocidade reprodutiva). No entanto, deve-se considerar que variações nos coeficientes "a" e "b" da relação peso-comprimento ocorreram não somente entre as espécies, mas também entre estoques da mesma espécie (Narahara et al., 1985).

Os resultados dos valores médios do fator de condição dos exemplares para os dois pontos amostrados estão representados na Figura 2. Segundo Vazzoler (1996), o fator de condição expressa o estado fisiológico do peixe, refletindo interações de fatores bióticos e abióticos, indicando condições de nutrição e variando de acordo com o ciclo de maturação sexual que, associado a outras evidências, indica o período de reprodução. Esse perfil de comportamento também foi observado para Prochilodus

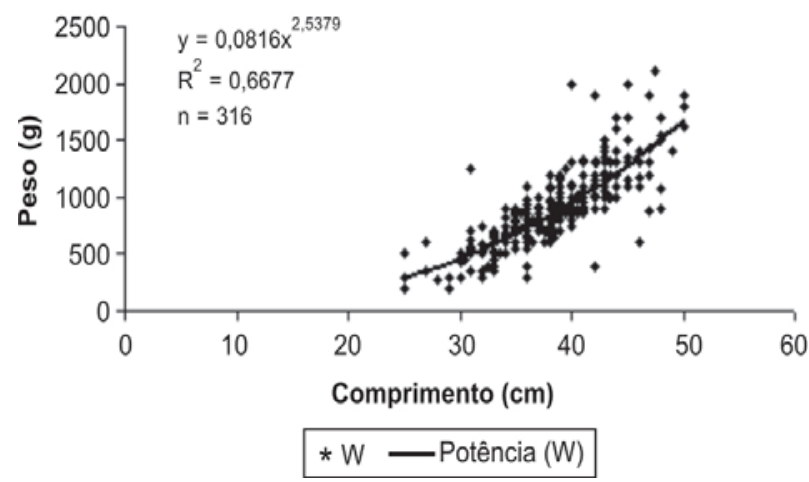

Figura 1. Relação peso-comprimento de curimbatá (Prochilodus lineatus) do rio Mogi-Guaçu coletado com tarrafa na barragem de Cachoeira de Emas e na foz do rio Jaguari-Mirim. scrofa (Vieira, 1984); Prochilodus nigricans (Braga, 1990); e Prochilodus cearensis (Araujo et. al., 2002).

Neste trabalho, foi verificado aumento gradual do fator de condição durante o período reprodutivo, decrescendo logo após o pico da reprodução. Os mais altos valores ocorreram em novembro, correspondentes ao pico de desova, e os menores, em março, possivelmente sinalizando o fim do período reprodutivo. Esses dados são indicativos de melhor condição fisiológica propícia para a reprodução da espécie.

Os valores do fator de condição (K) observados no período de inverno significam que os exemplares encontravam-se em melhores condições na Foz do Jaguari-Mirim, possivelmente em decorrência de maior disponibilidade de alimento nesse ambiente e também pelos valores de IGS (Figuras 3 e 4), embora não tão expressivos, contudo maiores do que os encontrados para exemplares capturados na barragem de Cachoeira de Emas para o referido período. Normalmente, altos níveis populacionais resultam em menor quantidade de alimento por peixe e baixo fator de condição, enquanto baixos níveis populacionais resultam em maior quantidade de alimento por peixe e alto fator de condição (Barbieri \& Verani, 1987). A constância de captura verificada nos locais de coleta permite supor que exemplares capturados fora do período de migração reprodutiva possam vir a ser indivíduos residentes. Segundo Morelli et al. (2007), cardumes residentes são encontrados durante o ano todo. Para Godoy (1975), o curimbatá pode ser capturado durante o ano todo, porém mais intensamente entre outubro e março, durante a migração reprodutiva. Entre abril e setembro são apanhadas quantidades bem menores e, ainda, constituídas de indivíduos de pequeno porte, pois com a migração trófica após as desovas os curimbatás, em maior parte, descem para o "lar de alimentação", situado no médio Rio Grande.

Portanto, é coerente pensar que esses peixes, em sendo residentes, possivelmente não metabolizam gordura o suficiente em função da não migração e, portanto, têm valores de $\mathrm{K}$ mais elevados.

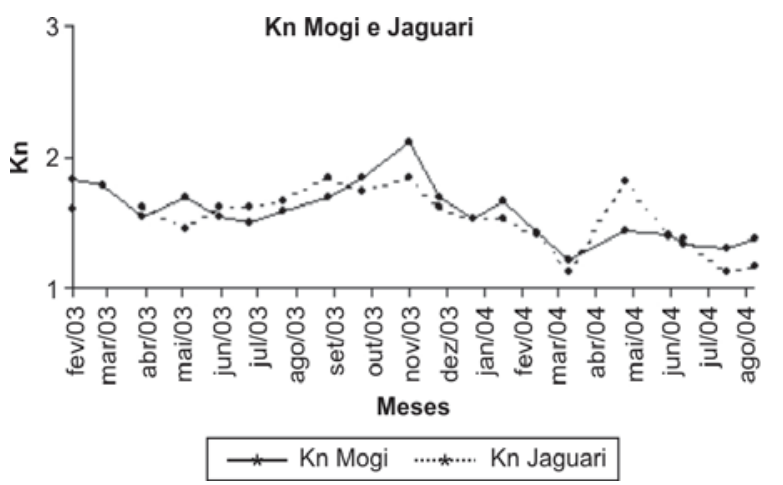

Figura 2. Fator de condição do curimbatá (Prochilodus lineatus) do rio Mogi-Guaçu capturado com tarrafa na barragem de Cachoeira de Emas e na foz do rio Jaguari-Mirim. 


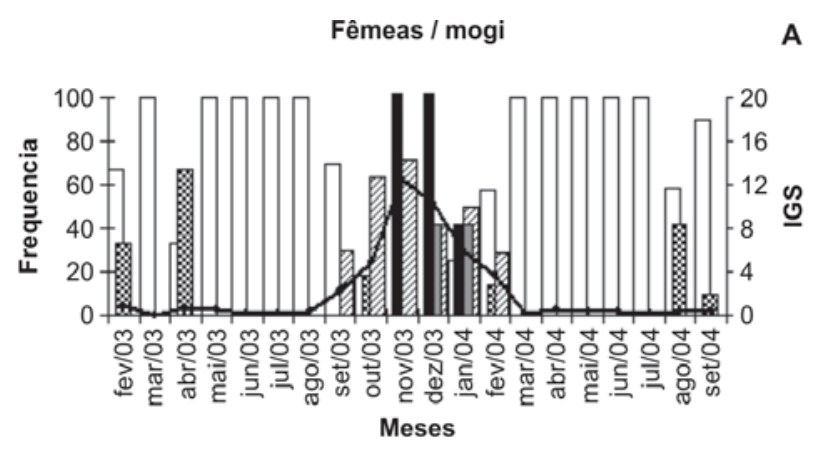

$\square$ repouso $\mathrm{m}$ emmat mIme panmad $\square$ maduro $\square$ esgotado $\rightarrow$ IGS

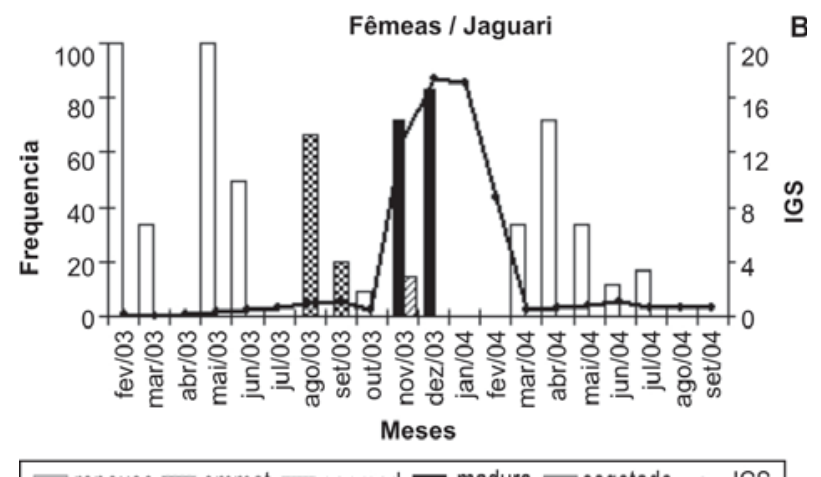

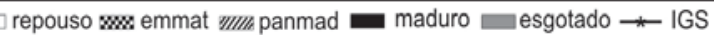

Figura 3. Índice gonados somático (IGS) e frequência de estádios de maturação gonadal de fêmeas de curimbatá (Prochilodus lineatus) do rio Mogi-Guaçu capturadas com tarrafa na barragem de Cachoeira de Emas (A) e na foz do rio Jaguari-Mirim (B).

As observações realizadas nos dois pontos de coleta para a variação temporal dos estádios de maturação gonadal, com base na distribuição de freqüência, e os valores de IGS (Figuras 3 a 4) evidenciam um período reprodutivo bem definido para o curimbatá entre os meses de outubro e janeiro, com maior atividade reprodutiva em novembro e dezembro, dados esses que corroboram observações de Barbieri et al. (2000), os quais afirmam ainda que esse período curto observado é característica de peixes de desova total. Portanto, os maiores valores de IGS ocorreram na época de maior captura de exemplares maduros e os menores, na pós-desova. Resultados semelhantes foram obtidos para Hypostomus commersoni por Agostinho et al. (1991) e Serrasalmus brandtii por Teles \& Godinho (1997).

Nos resultados, foi observada também elevada frequência de machos maduros no mês de setembro, caracterizando antecipação das fêmeas no processo de maturação gonadal (Figuras 3B e 4B) para os dois pontos amostrados. A proporção sexual de curimbatá foi de 1:1 na barragem de Cachoeira de Emas, exceto para o mês de setembro de 2004, quando houve prevalência de fêmeas, e na foz do rio Jaguari-Mirim também apresentou o mesmo comportamento, ou seja 1:1, e houve prevalência de fêmeas somente nos meses de janeiro e março de 2004 (Figuras 5A e 5B). Esses resultados corroboram as obser-
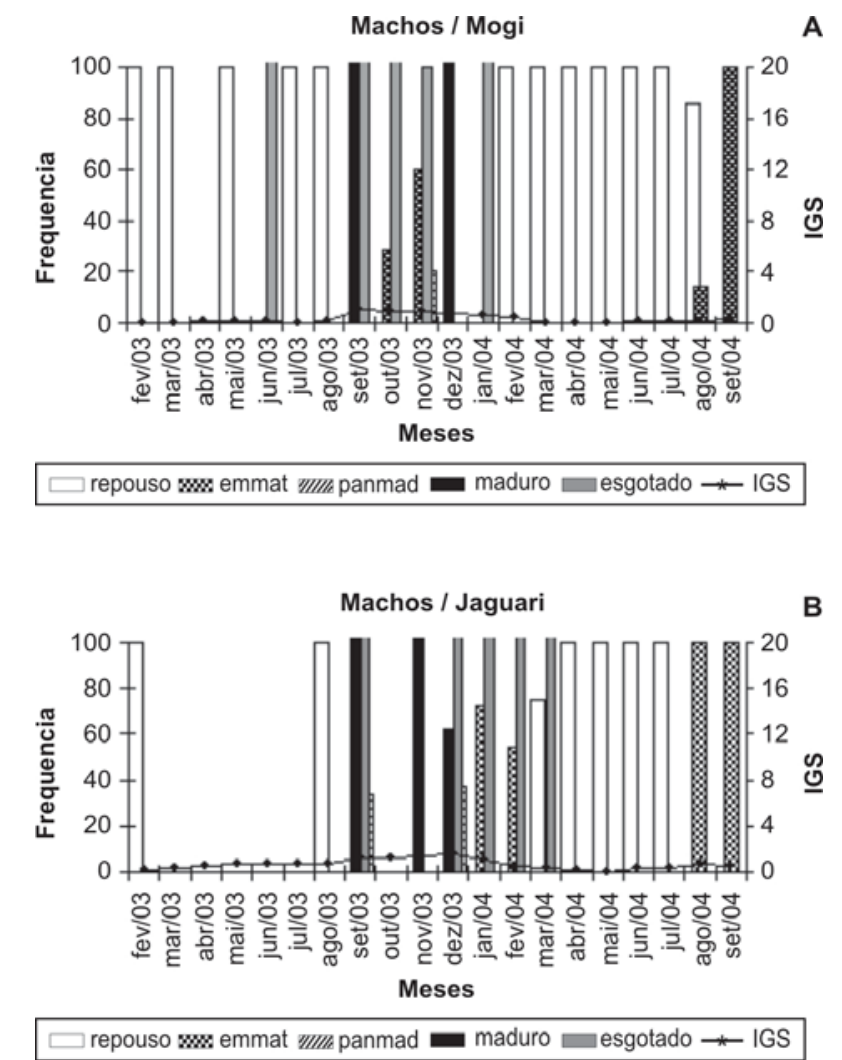

Figura 4. Índice gonados somático (IGS) e frequência de estádios de maturação gonadal de machos de curimbatá (Prochilodus lineatus) do rio Mogi-Guaçu capturados com tarrafa na Barragem de Cachoeira de Emas (A) e na foz do rio Jaguari-Mirim (B).

vações de Vazzoler (1996), o qual afirma que a proporção sexual em peixes varia ao longo do ciclo de vida em função de eventos sucessivos, que atuam de modo distinto sobre os indivíduos de cada sexo, sendo, na maioria dos casos, a proporção 1:1 observada para a população como um todo. Outros autores também encontraram essa proporção, como os trabalhos apresentados por Dias et al. (2004) com Pinirampus pinirampu, Santos et al. (2004) com Orthopristis ruber e Silva et al. (2005) com Scomberomorus brasiliensis. Por outro lado, Resende et al. (1995) encontraram predominância de machos de curimbatá no rio Miranda, MS, enquanto Araujo \& Gurgel (2002) relataram proporção de 3:1 (fêmea/macho) para Prochilodus cearencis no açude Itans, Caicó, RN, e Shibatta (2005) encontrou proporção de fêmeas mais elevada que a de machos para Simpsonichthys boitone, na Reserva Ecológica do IBGE em Brasília, DF.

Curimbatá foi mais abundante na barragem de Cachoeira de Emas do que na foz do Jaguari-Mirim (Figuras 6 e 7), apresentando picos de CPUE entre agosto e outubro, com os valores mais baixos encontrados em maio, junho e julho. Os referidos picos para curimbatá correspondem aos de migração (setembro/outubro) observados por Capeleti \& Petrere Jr. (2006) na barragem de Cachoeira de Emas. Os autores mencionam ainda que os últimos cardu- 

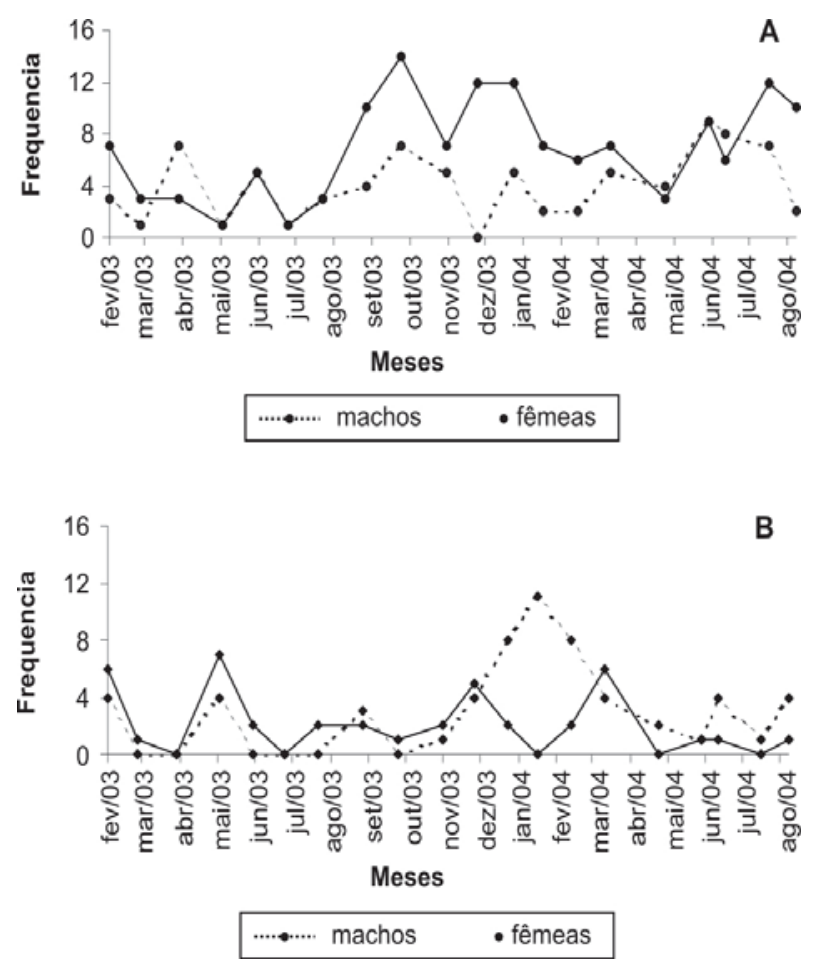

Figura 5. Proporção sexual de curimbatá (Prochilodus lineatus) na Barragem de Cachoeira de Emas (A) e na Foz do rio JaguariMirim (B), no rio Mogi - Guaçu.

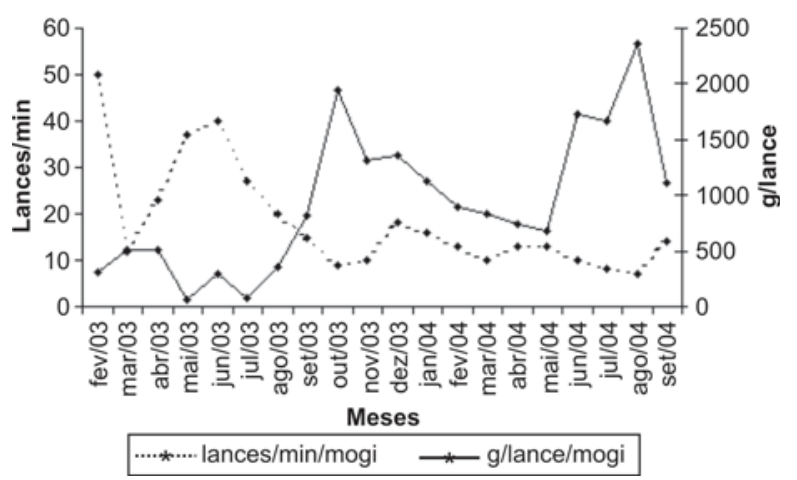

Figura 6. Distribuição mensal de CPUE do curimbatá na Barragem de Cachoeira de Emas de fevereiro de 2003 a setembro de 2004.

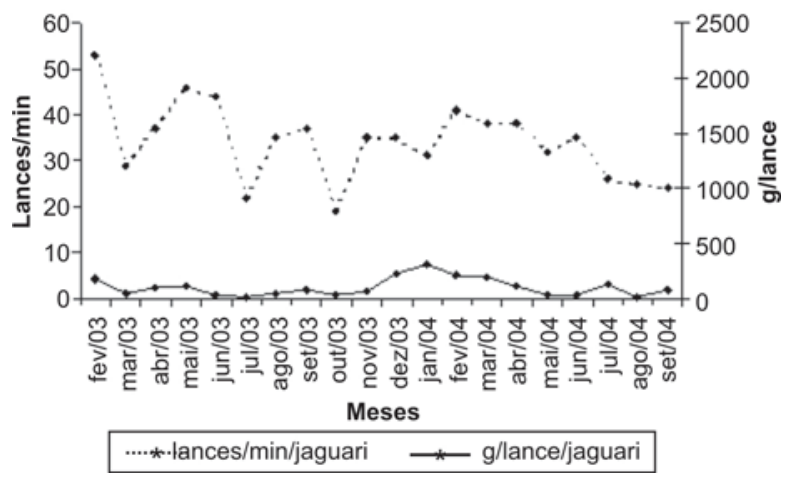

Figura 7. Distribuição mensal de CPUE do curimbatá na Foz do rio Jaguari-Mirim de fevereiro de 2003 a setembro de 2004. mes chegam na barragem de Cachoeira de Emas em março e abril, regionalmente chamados de temporão, e apresentam comprimento médio inferior e estádios não avançados de maturação.

\section{CONCLUSÕES}

O crescimento dos indivíduos adultos de curimbatá do rio Mogi-Guaçu é do tipo alométrico negativo

O período de reprodução de curimbatá ocorre entre os meses de outubro e janeiro, com picos nos meses de novembro e dezembro

Os machos de curimbatá antecipam a maturação gonadal em relação às fêmeas

No período reprodutivo a proporção sexual de curimbatá no rio Mogi-Guaçu é de 1:1.

\section{REFERÊNCIAS BIBLIOGRÁFICAS}

Agostinho AA, Hahn NS \& Agostinho CS (1991) Ciclo reprodutivo e primeira maturação de fêmeas de Hypostomus commersoni (Valenciennes,1840) (Siluriformes, Loricariidae) no revervatório Capivari-Cachoeira-PR. Revista Brasileira de Biologia, 51:31-37.

Araujo FG, Duarte S, Goldberg RS \& Fichberg I (2000) Ciclo reprodutivo de Parauchenipterus striatulus (PiscesAuchenipteridade) na represa de Ribeirão das Lajes -RJ. Arquivo Brasileiro de Medicina Veterinaria e Zootecnia, 52:276-284.

Araujo de AS \& Gurgel HCB (2002) Aspectos da biologia de Prochilodus cearensis (Steindachner, 1911) (Characiformes, Prochilodontidae) no açude Itans/Caicó, Rio Grande do Norete, Brasil. Acta Scientiarum Biological Sciences, 4:85-96.

Barbieri G \& Verani JR (1987).O fator de condição como indicador do período de desova em Hypostomus aff. Plecostomus (Linnaeus, 1758) (Osteichthyes, Loricariidae) na represa do Monjolinho (São Carlos, SP). Ciência e Cultura, 39:655-658.

Barbieri G, Salles AF \& Cestarolli MA (2000) Análise populacional do curimbatá Prochilodus lineatus, do rio Mogi-Guaçu, Pirassununga/SP (Characiformes, Prochilodontidade). Boletim do Instituto de Pesca, 26:137-145.

Braga FMS (1986) Estudo entre fator de condição e relação peso/ comprimento para alguns peixes marinhos. Revista Brasileira de Biologia, 46:339-346.

Braga FMS (1990) Aspectos da reprodução e alimentação de peixes comuns em um trecho do rio Tocantins entre Imperatriz e Estreito, Estados do Maranhão e Tocantins, Brasil. Revista Brasileira de Biologia, 50:547-558.

Capeleti AR \& Petrere Jr M (2006) Migration of the curimbatá Prochilodus lineatus (Valenciennes, 1836) (Pisces, Prochilodontidae) at the waterfall "Cachoeira de Emas" of the Mogi-Guaçu River-São Paulo, Brasil. Brazilian Journal Biological, 66:651-659.

Costa APR, Andrade DR, Vidal MVJ \& Souza G (2005) Indicadores quantitativos da biologia reprodutiva de fêmeas de piau-vermelho no Rio Paraiba do Sul. Pesquisa Agropecuaria Brasileira, 40:789-795.

Dias JH, Britto SGC, Vianna NC \& Garavello J C (2004) Biological and ecological aspects of Pinirampus pirinampu (Spix, 1829) Siluriformes, Pimelodidae, in Capivara reservoir, Paranapanema River Southern Brazil. Acta Limnologica Brasileira, 16:293-304. 
Godoy MP (1975) Peixes do Brasil: subordem Characoidei. Piracicaba, Franciscana, v. IV. p631-847.

Gomiero LM \& Braga FMS (2003) Relação peso-comprimento e fator de condição para Cichla cf. ocllaris e Cichla monoculus (Perciformes, Cichlidae) no reservatório de Volta Grande, rio Grande-MG/SP. Maringá, 25:79-86.

Goulart E \& Verani JR (1992) Proporção sexual, relação peso/ comprimento e fator de condição de Hypostomus commersonii Valenciennes, 1840 (Osteichthyes Loricariidae) da represa Capivari-Cachoeira, Paraná, Brasil. Revista UNIMAR, 14(suplemento):019-033.

Gurgel, HCB; Barbieri G \& Verani JR (1997) Análise do fator de condição de Metynnis cf roosevelti Eigenmann, 1915 (Characidae, Myleinae) da lagoa Redonda, Município de Nísia Floresta, Rio Grande do Norte, Brasil. IN: VIII Seminário Regional de Ecologia, São Carlos. Anais, UFSCar. p.357-376.

Gurgel HCB (2004) Estrutura populacional e época de reprodução de Astyanax fasciatus (Cuvier) (Characidae, Tetragonopterinae) do Rio Ceará Mirim, Poço Branco, Rio Grande do Norte. Revista Brasileira de Zoologia, 21:131-135.

Le Cren ED (1951) The lenght-weight relationship and seasonal cycle in gonad weight and condition in perch Perca fluviatilis. Journal Animal Ecology, 20:201-219.

Morelli KA, Oliveira C, Porto-Foresti F, Senhorine JA \& Forest F (2007) Population structure of Prochilodus lineatus (Characiformes, Prochilodontidae) in the Mogi-Guaçu river identified by molecular analysis. Pp7-12. In: Pompeu PS, Santos HA \& Alves CBM (Eds.). Proceedings of the Symposium on Fish Passages in South America. Lavras, UFLA. 100p.

Narahara MY, Godinho HM, Fenerich-Verani N \& Romagosa E (1985) Relação Peso-comprimento e fator de condição de Rhamdia Hilarii (Valenciennes,1840) (Osteichthyes, Siluriformes, Pimelodidae). Boletim Instituto de Pesca, 12:13-22.

Orsi ML, Shibata AO \& Silva-Souza AT (2002) Caracterização biológica de populações de peixes do rio Tibagi, localidade de Sertanópolis, p 425-432. In: M.E. Medri (Ed). A bacia do Rio Tibagi. Londrina, Universidade Estadual de Londrina, 595p.

Orsi ML, Carvalho ED \& Foresti F (2004) Biologia populacional de Astyanax altiparane Garutti \& Britiski (Teleostei, Characidae) do médio Rio Paranapanema, Paraná, Brasil. Revista Brasileira de Zoologia, 21:207-218.
Resende EK, Catella AC, Nascimento FL, Palmeira SS, Pereira RAC, Lima MS \& Almeida VLL (1995) Biologia do curimbatá (Prochilodus lineatus), pintado (Pseudoplatystoma corruscans) e cachara (Pseudoplatystoma fasciatum) na bacia hidrográfica do rio Miranda, Pantanal do Mato Grosso do Sul, Brasil. Corumbá, EMBRAPA-CPAP. 75p.

Santos ALB, Pessanha ALM, Costa MR \& Araujo FG (2004) Relação peso-comprimento de Orthopristis ruber (Cuvier) (Teleostei, Haemulidae) na Baia de Sepetiba, Rio de Janeiro, Brasil. Revista Brasileira de Zoologia, 21:185-187.

Silva GC, Castro ACL \& Gubiani EA (2005) Estrutura populacional e indicadores reprodutivos de Scomberomorus brasiliensis Collette, Russo e Zavala-Camin, 1978 (Perciformes: Scombridae) no litoral ocidental maranhense. Acta Science Biological Science, 27:383-389.

Shibatta OA (2005) Reprodução do pirá-brasília, Simpsonichthys boitonei Carvalho (Cyprinodontiformes, Rivulidae), e caracterização de seu habitat na Reserva Ecológica do Instituto Brasileiro de Geografia e Estatística, Brasília, Distrito Federal, Brasil. Revista Brasileira de Zoologia, 22:1146-1151.

Sokal RR \& Rohlf FJ (1981) Biometry: the principle and practice of statistics in biological research. New York, W.H. Freeman. 859p.

Teles MEO \& Godinho HP (1997) Ciclo reprodutivo da pirambeba Serrasalmus brandtii (Teleostei, Characidae) na represa de três marias, Rio São Francisco. Revista Brasileira de Biologia, 2:177184.

Verani JR (1980) Controle populacional em cultivo intensivo comparado entre a tilápia do Nilo Sarotherodon niloticus (Linnaeus,1757) e o tucunaré comum, Cichla ocellaris Schneider, 1801. Aspectos quantitativos. Dissertação de Mestrado. Universidade Federal de São Carlos, São Carlos. 116p.

Vianna M \& Verani JR (2002) Biologia populacional de Orthopristis ruber (Teleostei, Haemulidae) espécie acompanhante da pesca de arrasto do camarão-rosa, no sudeste brasileiro. Atlântica, 23:27-36.

Vieira AL (1984) Aspectos do metabolismo lipídico do curimbatá Prochilodus scrofa (Steindachner, 1881). Boletim do Instituto de Pesca, 11:63-68.

Vazzoler AEAdeM (1996) Biologia da reprodução de peixes teleósteos: teoria e prática. Maringá, EDUEM. 196p. 